\title{
Surgical technique of a recurrent post-radiation vesicovaginal fistula with a small intestine graft
}

\author{
Martin Vaso $^{1} \cdot$ Cornelia Betschart $^{1}$ (D) Herwig Egger $^{2} \cdot$ Daniel Fink $^{1} \cdot$ \\ Ana-Maria Schmidt ${ }^{1}$
}

Received: 24 December 2014/ Accepted: 11 May 2015/Published online: 19 May 2015

(C) Springer-Verlag Berlin Heidelberg 2015

\begin{abstract}
Vesicovaginal fistulas are devastating conditions for the affected women. The combination of a hysterectomy and radiation increases the fistula risk 5-10 times. Radiation-induced recurrent vesicovaginal fistulas have the lowest success rate and require the most demanding treatment. We present the case of a recurrent postradiation vesicovaginal fistula treated with a small intestine graft after unsuccessful conservative and failed previous operative treatments. The surgical management with a small intestine graft led to a permanently closed fistula. We describe the surgical abdominal procedure step-wise and review the rather scarce, post-radiation fistula literature. The closure of a vesicovaginal fistula with a small intestine graft is a complex surgical treatment with a long-term, successful result.
\end{abstract}

Keywords Recurrent vesicovaginal fistula - Intestinal graft $\cdot$ Post-radiation $\cdot$ Complication $\cdot$ Abdominal procedure cervical cancer

Cornelia Betschart

cornelia.betschart@usz.ch

Martin Vaso

martin.vaso@gmail.com

Herwig Egger

herwig.egger@t-online.de

Daniel Fink

daniel.fink@usz.ch

Ana-Maria Schmidt

ana-maria.schmidt@usz.ch

1 Department of Gynecology, University Hospital Zurich, Frauenklinikstrasse 10, 8091 Zurich, Switzerland

2 Department of Obstetrics and Gynecology, Clinic Neumarkt, Neumarkt, Germany

\section{Introduction}

The physical, mental and social implications of a vesicovaginal fistula are stressful for the affected women [1]. In developed countries, hysterectomy is the most common cause of vesicovaginal fistula $[1,2]$. The combination of a hysterectomy and radiation increases the fistula risk 5-10 times [3]. Various treatment options with different success rates are available. Recurrent post-radiation, vesicovaginal fistula has the lowest success rate and requires the most demanding treatment [4]. We performed a closure of a recurrent vesicovaginal fistula with a small intestine graft that lead to a permanent closure of the fistula. This procedure has been performed by Mraz more than 20 years ago. We aim to give a detailed description of this surgical technique, that up to now is not available in an electronic or open-access journal [5].

\section{Case presentation}

A 43-year-old patient, suffering from a squamous cell carcinoma of the cervix (FIGO staging IIIb) underwent radiochemotherapy $\left(50 \mathrm{mg} / \mathrm{m}^{2}\right.$ cisplatin weekly) for six cycles. The radiochemotherapy had to be halted because of radiation-induced injury to the bowel. Hence, the patient underwent a radical extended hysterectomy (Piver classification II-III) with en bloc resection of the distal left ureter, pelvic lymphadenectomy and reimplantation of the left ureter with a psoas-hitch plasty. The patient's personal history included cannabis and nicotine abuse of $>20$ py and the resection of an adenosquamous carcinoma of the right upper lobe of the lung (pT2 pN0 G3 R0) 5 years prior. The patient had a cachectic habitus (BMI $17 \mathrm{~kg} / \mathrm{m}^{2}$ ). Postoperatively, a vesicovaginal fistula developed. Initially, 
Fig. 1 a Preparation of the oral part of the ileum $(12 \mathrm{~cm}$ in length) leading to the previous ileostomy with an intact vascular pedicle; $\mathbf{b}$ the dissected preparation of the small intestine was opened antimesenterically to obtain a rectangular graft and cleaned; c the intestinal flap was sewn tension-free to the bladder margins with continuous suture
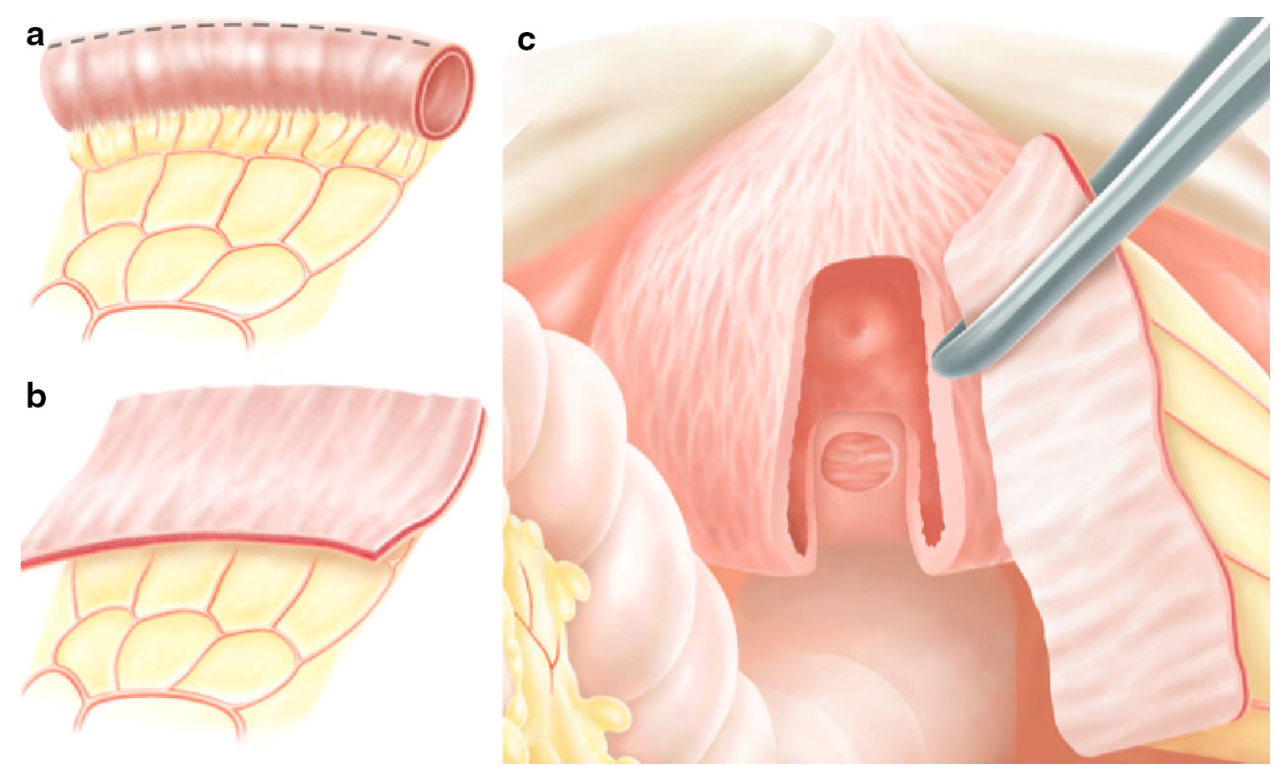

conservative treatment with a permanent suprapubic catheter and intramuscular estrogenic depot showed positively affected continence, but this effect was not permanent. After failed conservative treatment, an abdominal surgical approach with a rectus abdominis flap was performed with ongoing suprapubic bladder drainage. Nine days postoperatively, the patient showed sepsis with a small bowel leakage and intestinal fluid in the drainages and the indwelling bladder catheter. The patient underwent a third laparotomy because of a small intestine perforation, and a protective ileostoma was installed $10 \mathrm{~cm}$ proximal to the ileocecum. Three weeks after the abdominal fistula occlusion, the vesicovaginal fistula reappeared and the intestinal flap technique for the closure of the fistula was performed.

\section{Abdominal occlusion of a vesicovaginal fistula with a seromuscular small intestinal graft after extreme adhesiolysis, relocation of the protective ileostomy, and ileocaecal pole resection with stapled anastomosis}

The previous longitudinal laparotomy was reopened up to two fingers above the umbilicus. Because of three antecedent laparotomies, and previous attempts to close the fistula including a protective ileostoma, an extended adhesiolysis was needed to liberate the cuff of the vagina from the bladder back wall and small intestine. To exclude a tumor recurrence, which would have halted further intervention, frozen sections of the peritoneum and serosa of the intestine were taken, and they showed no signs of malignancy. The bladder preparation was particularly complex because of adhesions and post-radiation fibrosis.
Next, the bladder was filled with saline and opened sagittally at the bladder dome. Two fistulas positioned sideby-side measuring 5 and $7 \mathrm{~mm}$ were found at approximately the same location as the previous fistula. In a classical approach, the separation of the bladder from the vagina could be performed successfully. Because of the firm, solid adhesions between the vaginal cuff and the rectum, a primary separation of the vagina from the rectum was not possible without a high risk of harming the rectum. The two fistulas were excised to receive fresh tissue margins with good blood supply.

Thereafter, the ileostoma was closed, cut out and mobilized. However, it became apparent that the remaining aboral portion of the small intestine behind the ileostoma was too short, and the blood circulation was insufficient. Therefore, it was inappropriate to use it as an interposition graft. The oral part of the ileum leading to the previous ileostomy was isolated after identifying the vascular pedicle by diaphanoscopy (Fig. 1a). This part of the small intestine was opened antimesenterically and cleaned (Fig. 1b). The vascular pedicle was long enough to reach the bladder. Subsequently, the intestinal flap was sewn tension-free to the bladder margins with continuous monofilament PDS 3-0 suture and served as a partial bladder wall replacement (Fig. 1c). The interposed small intestine remained rosy and well supplied with blood. Filling the bladder with $100 \mathrm{ml}$ of methylene blue revealed a slight leakage that was resolved with a Z-suture.

The fistula in the vagina could not be closed primarily because of its adhesion to the rectum. It was covered with the back wall of the intestinal flap and fixed with three PDS stitches to eliminate the possibility of the abdominal cavity opening to the vaginal environment. 


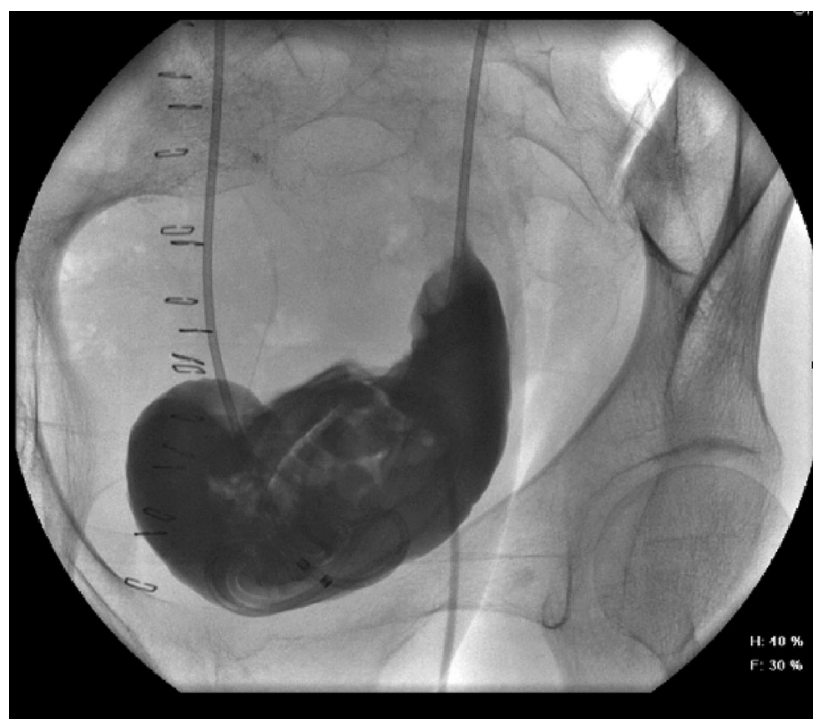

Fig. 2 Cystogram confirms the integrity of the repair

Then, a stapled ileum-caecum-pole resection with an ileum-ascending colostomy was performed, with follow-up suture. A Jackson-Pratt drain was left in the small pelvis and also along the anastomosis of the intestine extending from the right side. The location site of the ileostomy in the abdominal wall was closed. The abdominal wall was sutured with Everett's method, subcutaneous PDS sutures, and surgical staples for the skin.

The blood loss was $500 \mathrm{ml}$, and the surgery time was $7 \mathrm{~h}$ and $23 \mathrm{~min}$.

Postoperatively, a suprapubic catheter was placed for 6 weeks, and prior to its removal, a retrograde cystogram (Fig. 2) was performed to confirm the integrity of the repair.

The later postoperative course was complication free, and the bladder function was well restored without any post-void residual volume or involuntary detrusor contractions. At the 4-year follow-up, no vesicovaginal fistula was detected.

\section{Discussion}

The treatment of vesicovaginal fistula can be categorized as operative and non-operative therapy. If small fistulas are diagnosed in the first few post-surgical weeks, non-surgical therapies, such as an indwelling urinary catheter $[6,7]$, the injection of fibrin glue [8-10] and the use of electro cautery [11] can resolve a minority of vesicovaginal fistulas. Surgery is undertaken when patients are not responding to non-operative therapy. Unsuccessful repairs occur in 3-25\% of surgery-caused fistulas; previous radiotherapy and recurrent fistula repairs have lower success rates
[12-14]. Surgical techniques can be applied transvaginally, transabdominally, transvesically or in a combination. Transvaginal surgery has a lower risk of complications, a lower blood loss and a faster recovery time [15], while the transabdominal approach is indicated for complex, recurrent or tall fistula $(>3 \mathrm{~cm})$ [16], and for highly supratrigonal [16] or fistula located near the ureteric ostium [1]. Furthermore, the transabdominal approach, which was described by O'Conner, has a higher success rate ( $>95 \%)$ than the transvaginal approach $[2,17]$. The use of interposition grafts is essential and promising for damaged and irradiated tissue of complex fistula, while its use for small fistula is still debatable [13]. Studies have described the use of the omentum, appendix of the sigmoid colon, gracilis muscle flap, rectus abdominis muscle flap, myocutaneous flap, myofascial flap and bladder mucosa as interposition grafts; however, none of these studies described the use of grafts in previously irradiated vesicovaginal tissue $[13,18$, 19].

Only one study met the criteria of a post-radiogenic recurrent fistula description. It was Mráz et al. [5] that treated between 1977 and 1988 four patients with radiogenic fistulas $(1.5-3 \mathrm{~cm}$ diameter) with the use of a seromuscular intestine interposition graft. As with our patient, the patients in that study were suffering from cervical carcinoma, and were primarily treated with radiotherapy and secondarily with radical hysterectomies. Only one of these four patients had a previous surgical attempt for fistula occlusion, which had failed. Postoperatively, two of four patients showed involuntary contractions of the bladder with clinical signs of bladder instability. Treatment with oxybutynin was administered successfully. The capacity of the bladder 6 months after surgery ranged between 220 and $280 \mathrm{ml}$, and it increased by $20 \%$ in the following 2 years. There was no leakage over the next 3.5-5 postoperative years [5].

Surgical treatment for a small intestine graft is particularly suitable for high-complex fistulas, such as fistulas that occur after radiotherapy and may result in small vessel endarteritis obliterans, an ongoing process that can result in a fistula occurring years later. These fistulas may be accompanied by vascular disruption with the potential of a recurring fistula. The intestinal flap has this advantage: a non-irradiated, well-vascularized tissue is placed in a high risk environment. Nevertheless, this treatment type requires highly specialized, interdisciplinary trained experts.

Acknowledgments We acknowledge the graphical support from Stefan Schwyter, medical illustrator at the University Hospital Zurich.

Conflict of interest The authors declare that they have no conflict of interest. 
Informed consent Written informed consent was obtained from the patient for publication of this case report and the accompanying cystography.

\section{References}

1. Garthwaite M, Harris N (2010) Vesicovaginal fistulae. Indian J Urol 26:253-256

2. Alonso y Gregorio S, Alvarez Maestro M, Cabrera Castillo PM, Hidalgo Togores L, de la Peña Barthel JJ (2009) Laparoscopic repair of the vesicovaginal fistula (laparoscopic O'Connor repair). Actas Urol Esp 33:1133-1137

3. Boronow RC (1986) Repair of the radiation-induced vaginal fistula utilizing the Martius technique. World J Surg 10:237-248

4. Haferkamp A, Wagener N, Buse S, Reitz A, Pfitzenmaier J, Hallscheidt P, Hohenfellner M (2005) Vesicovaginal fistulas. Urologe A 44:270-276

5. Mráz JP, Sutorý M (1994) An alternative in surgical treatment of post-irradiation vesicovaginal and rectovaginal fistulas: the seromuscular intestinal graft (patch). J Urol 151:357-359

6. Davits RJ, Miranda SI (1991) Conservative treatment of vesicovaginal fistulas by bladder drainage alone. $\mathrm{Br} \mathrm{J}$ Urol 68:155-156

7. Petri E, Alken P (2000) Gynäkologische Urologie: Aspekte der interdisziplinären Diagnostik und Therapie. 3rd edition, Thieme, Stuttgart

8. Pettersson S, Hedelin H, Jansson I, Teger-Nilsson AC (1979) Fibrin occlusion of a vesicovaginal fistula. Lancet 1:933
9. Sharma SK, Perry KT, Turk TM (2005) Endoscopic injection of fibrin glue for the treatment of urinary-tract pathology. J Endourol 19:419-423

10. D'Arcy FT, Jaffry S (2010) The treatment of vesicovaginal fistula by endoscopic injection of fibrin glue. Surgeon 8:174-176

11. Stovsky MD, Ignatoff JM, Blum MD, Nanninga JB, O’Conor VJ, Kursh ED (1994) Use of electrocoagulation in the treatment of vesicovaginal fistulas. J Urol 152:1443-1444

12. Sotelo R, Moros V, Clavijo R, Poulakis V (2012) Robotic repair of vesicovaginal fistula (VVF). BJU Int 109:1416-1434

13. Smith GL, Williams G (1999) Vesicovaginal fistula. BJU Int 83:564-569 (quiz 9-70)

14. Sjøveian S, Vangen S, Mukwege D, Onsrud M (2011) Surgical outcome of obstetric fistula: a retrospective analysis of 595 patients. Acta Obstet Gynecol Scand 90:753-760

15. Angioli R, Penalver M, Muzii L, Mendez L, Mirhashemi R, Bellati F, Crocè C, Panici PB (2003) Guidelines of how to manage vesicovaginal fistula. Crit Rev Oncol Hematol 48:295-304

16. Hemal AK, Kolla SB, Wadhwa P (2008) Robotic reconstruction for recurrent supratrigonal vesicovaginal fistulas. $J$ Urol 180:981-985

17. O'Conor VJ (1980) Review of experience with vesicovaginal fistula repair. J Urol 123:367-369

18. Woo HH, Rosario DJ, Chapple CR (1996) The treatment of vesicovaginal fistulae. Eur Urol 29:1-9

19. Zhang Q, Ye Z, Liu F, Qi X, Shao C, He X, Zhang D (2012) Laparoscopic transabdominal transvesical repair of supratrigonal vesicovaginal fistula. Int Urogynecol J 24(2):337-342 